\title{
Cryo-SiN - An Alternative Substrate to Visualize Active Viral Assemblies
}

Keywords: Cryo-SiN; Silicon nitride; Affinity capture; Cryo-Electron Microscopy; Rotavirus

\begin{abstract}
Silicon nitride ( $\mathrm{SiN}$ ) membranes that are merely nanometers-thick have been introduced as new support films for electron microscopy (EM) applications. The use of these thin membranes for high-resolution imaging is widely attractive due to their consistent manufacture, flatness, and durability. Here, we utilized SiN support films to actively monitor key steps in rotavirus RNA synthesis at the nanoscale. Rotaviruses are gastrointestinal pathogens that infect pediatric populations and pose a major health problem in developing countries. We demonstrate, for the first time, that cryo-EM specimens of these viral assemblies may be prepared using SiN membranes, that we have termed "cryo-SiN". Cryo-SiN microchips containing inactive and actively transcribing rotavirus complexes were vitrified using gentleblot conditions to yield reproducibly thin ice-embedded specimens. EM imaging results indicate that cryo-SiN samples exhibited greater visual contrast than specimens prepared on conventional support films under the same conditions. The improved contrast of the cryo-SiN samples permitted us to calculate 3D structures of rotavirus complexes using a fraction of the images required for conventionally prepared specimens. We anticipate the use of cryo-SiN microchip will enable us to better investigate critical viral life-cycle processes at the molecular level under near-native conditions.
\end{abstract}

\section{Abbreviations}

Double-Layered Particles (DLPs); Silicon Nitride (SiN); Electron Microscopy (EM); Transmission Electron Microscope (TEM); Nickel-Nitrilotriacetic Acid (Ni-NTA); Nanometers (nm)

\section{Introduction}

Macromolecules orchestrate processes to maintain cells and tissues, having a critical role in biomedical research. Transmission electron microscopes (TEM) are used to illuminate the structural complexities of biological assemblies for interpreting molecular mechanisms. The use of innovative new materials in the electron microscopy (EM) field may advance current practices to view biological structures and to understand their related functions. Amorphous carbon is the conventional support material used to prepare biological specimens for cryo-EM structure determination. Micron-sized holes produced in carbon support films provide a transparent view through select regions in the film, creating the ideal background for imaging weak-phase objects such as biological assemblies [1].

One inherent limitation in the use of holey carbon films is beam-induced movement that results from charging effects [2]. To attenuate charging effects, specimens can also be prepared on holey carbon films having an added layer of amorphous carbon that covers the perforated film. More recently, novel support films have been

\section{Journal of}

\section{Analytical \& Molecular Techniques}

\author{
Justin R. Tanner ${ }^{1}$, Andrew C. Demmert ${ }^{2}$, Madeline \\ J. Dukes ${ }^{3}$, Linda A. Melanson ${ }^{4}$, Sarah M. \\ McDonald $^{1,2}$ and Deborah F. Kelly ${ }^{1,2 *}$ \\ ${ }^{\prime}$ Virginia Tech Carilion Research Institute, Roanoke, VA 24016 \\ ${ }^{2}$ Virginia Tech Carilion School of Medicine, Roanoke, VA 24016 \\ ${ }^{3}$ Applications Science, Protochips, Inc., Raleigh, NC 27606 \\ ${ }^{4}$ Life Sciences Division, Gatan, Inc., Pleasanton, CA 94588 \\ Address for Correspondence
}

Deborah F. Kelly, Virginia Tech Carilion Research Institute, 2 Riverside Circle, Roanoke, VA 24016, USA, Tel: +1-540-526-2031; Fax: +1-540985-3373; Email: debkelly@vt.edu

Submission: 01 November 2013

Accepted: 04 December 2013

Published: 09 December 2013

Reviewed \& Approved by: Dr. Peng Xi

Department of Biomedical Engineering at Peking University, China

developed in an effort to mitigate beam-related movements during the imaging process. Among these new substrates are conductive materials composed of titanium-silicon metal glass (Ti88Si12) [3] and silicon carbide (cryomesh) [4]. Both of these substrates exhibited decreased charging effects in images of frozen-hydrated specimens. Alternatively, ultrathin membranes composed of pure silicon or silicon nitride $(\mathrm{SiN})$, materials commonly used in the semi-conductor industry, have been introduced as new substrates to prepare specimens for high-resolution EM. Employing these new substrates, researchers have imaged unstained DNA origami complexes [5], nanoparticles [6], and graphene [7] at near-atomic resolution. In fact, using silicon membranes, the first electron tomogram to achieve atomic resolution was recently recorded for unstained nanoparticle material [8]. In general, electron tomographic reconstructions require very flat substrates and drift-free conditions to enable the proper alignment between recorded image frames. Tilt-series collected on SiN membranes at high angles $\left( \pm 83^{\circ}\right)$ have provided the only $3 \mathrm{D}$ tomographic information recorded at the atomic level [8]. These results suggest that SiN membranes may provide superior flatness to conventionally produced carbon substrates. Taken together, these exciting results suggest that silicon-based materials may hold great potential for preparing unstained cryo-electron microscopy (EM) specimens for structural biology experiments.

Our previous work demonstrated the feasibility to isolate a variety of specimens onto carbon-based support films for EM imaging of negatively stained and frozen-hydrated samples $[9,10]$. We have also recently examined rotavirus double-layered particles (DLPs) as negatively stained specimens using SiN membranes [11]. Continuing this line of investigation, here we explore the use of SiN microchips to prepare cryo-EM specimens of actively transcribing DLPs (Figure 1). In this context, we refer to these silicon nitride support membranes as "cryo-SiN". The use of cryo-SiN to prepare single particle EM samples is widely attractive due to the consistent manufacture of the nanometer-thin membranes. As the production of carbon 
support films can be highly variable, surfaces that are invariably flat, impermeable, and transparent to the electron beam would provide a valuable tool to the EM community. Here, we demonstrate that rotavirus DLPs can be readily captured on cryo-SiN in an active state, then vitrified and visualized directly by single particle cryo-EM.

\section{Materials and Methods}

\section{DLP preparation}

Rotavirus (strain SA11-4F) DLPs were prepared as described previously [12]. For transcriptional activation reactions, 25- $\mu \mathrm{l}$ mixtures were produced in eppendorf tubes containing $1 \mu \mathrm{g}$ DLPs, $100 \mathrm{mM}$ Tris- $\mathrm{HCl} \mathrm{pH} \mathrm{7.5,} 6 \mathrm{mM} \mathrm{MgAc}, 4 \mathrm{mM}$ DTT, $2 \mathrm{mM}$ each of ATP, GTP, CTP, UTP, $1 \mu l$ RNasin (Promega Corp., Madison, WI). The reactions proceeded for 30 minutes at $37^{\circ} \mathrm{C}$ prior to performing Affinity Capture experiments.

\section{Affinity grids and cryo-SiN preparation}

Affinity grids or silicon nitride microchips containing Nickelnitrilotriacetic acid (Ni-NTA) coatings were prepared as described previously [11]. The Ni-NTA functionalized lipid layers and each subsequent protein component were added to the front side of the $\mathrm{SiN}$ microchips. Aliquots $(3-\mu \mathrm{l})$ of His-tagged Protein A $(0.01 \mathrm{mg} /$ $\mathrm{mL}$ ) (Abcam, Cambridge, MA) in buffer solution containing $50 \mathrm{mM}$ Hepes, $\mathrm{pH} 7.5,150 \mathrm{mM} \mathrm{NaCl}, 10 \mathrm{mM} \mathrm{MgCl}_{2}$ and $10 \mathrm{mM} \mathrm{CaCl}_{2}$ were incubated for 1 minute on each holey carbon grid (C-flat $-2 / 1$ holey carbon grids; Protochips, Inc. Raleigh, NC) or SiN microchips (20 nm-thick SiN film, 9 windows; TEMwindows, West Henrietta, NY; and 30-nm thick DuraSiN, single window; Protochips, Inc.) coated with the Ni-NTA layer. The excess solution was blotted away and 3 - $\mu$ l aliquots of VP6-specific guinea pig polyclonal antisera (\#53963) $(0.01 \mathrm{mg} / \mathrm{ml}$ in the same buffer as Protein A) were added to Protein A-decorated substrates. Following 1-minute incubation, the antibody solution was removed using a Hamilton syringe and purified DLPs (2$\mu \mathrm{l}$ aliquots of $0.1 \mathrm{mg} / \mathrm{ml}$ ) that were either inactive or transcriptionally active were added to the substrates for a 2-minute incubation step. Negatively stained specimens were prepared as previously described $[10,11]$. Frozen-hydrated specimens were prepared using a Gatan Cryoplunge $^{\text {tw }} 3$ (Gatan, Inc., Pleasanton, CA) using one-sided blotting for approximately 8 seconds prior to plunging into liquid ethane.

\section{Electron Microscopy}

Frozen-hydrated EM Affinity grids and cryo-SiN specimens were transferred to a Gatan 626 cryoholder (Gatan, Inc.) and maintained under liquid nitrogen at $-180^{\circ} \mathrm{C}$ until inserted into the TEM. All specimens were imaged using a FEI Tecnai Spirit BioTwin TEM (FEI, Co., Hillsboro, OR) equipped with a LaB6 filament and operated at an acceleration voltage of $120 \mathrm{kV}$ under low-dose conditions $(\sim 5$ electrons / A22). Images of frozen-hydrated specimens were recorded on a FEI Eagle 2k HS CCD camera having a pixel size of $30-\mu \mathrm{m}$ using defocus values ranging from $-1.0 \mu \mathrm{m}$ to $-3.0 \mu \mathrm{m}$ at a nominal magnification of $50,000 \times$ for a final sampling of $6 \AA$ / pixel at the specimen level. Similarly, negatively stained specimens were imaged at varying magnifications $(4800 \times-50,000 \times)$.

\section{Image processing and $3 \mathrm{D}$ reconstruction}

Using the WEB interface associated with the SPIDER software package [13], 572 particles were selected from images of inactive DLP specimens prepared on vitrified holey carbon Affinity grids and 38 particles were selected from vitrified cryo-SiN microchips that were functionalized in the same manner. From images of actively transcribing DLPs prepared at the same time and under the same freezing conditions, we selected 192 particles from vitrified holey carbon Affinity grids and 100 particles from vitrified cryo-SiN microchips. Selected particle images were imported as separate image stacks into the RELION software package [14], used to calculate $3 \mathrm{D}$ structures of the non-transcribing and transcribing DLPs. A reference map for the inactive DLP structure [15] was downloaded from the website of the Grigorieff laboratory at Brandeis University. Implementing the RELION program, 3D reconstruction and refinement parameters included a pixel size of $6 \AA$, the reference model low-pass filtered to $80 \AA$, and a regularization parameter of $\mathrm{T}=4$, while enforcing icosahedral symmetry over an angular search space of 7.5 degrees.

\section{Results}

\section{Cryo-SiN specimens are easily imaged using low-dose TEM}

We first assessed the feasibility of using SiN microchips with the Affinity Capture system starting Parenthesis is missing before (Figure 1). SiN microchips (TEMwindows, SiMPore Inc. and Protochips, Inc.) were functionalized with Ni-NTA lipid layers on the front side of the chips followed by molecular adaptors, protein A and IgG antibodies against the VP6 protein found on the DLP capsid [12]. Negatively stained specimens were prepared and examined using TEM. Consistent with our previous findings [11], DLPs prepared in the presence of IgG antibodies can be readily recruited to the SiN surface, however, specimens lacking the antibody coating recruited little to no DLPs (Figure 1). As SiN microchips are hydrophobic in nature, they provide an ideal substrate to promote interactions with the lipid tail groups that are exposed at the air-water interface in the Ni-NTA lipid layers. Representative TEM images were recorded at various magnifications to indicate the level of specimen coverage on the $\mathrm{SiN}$ microchips (Figure 2). Based on these findings, we prepared frozen-hydrated specimens of DLPs using both functionalized holey carbon grids (Figure 3A) and cryo-SiN microchips (Figure 3B) by plunge freezing DLP samples into liquid ethane using a Gatan Cryoplunge $^{\mathrm{mx}} 3$ device with Gentle-Blot technology. Each specimen was prepared under the same conditions of $100 \%$ relative humidity at $25^{\circ} \mathrm{C}$ using 1 -sided blotting for $\sim 8$ seconds. Frozen-hydrated specimens were transferred to a 626 cryo specimen holder (Gatan, Inc.) and maintained under liquid nitrogen until transferred to the TEM.

We collected images of frozen-hydrated DLP specimens using a FEI Tecnai Spirit BioTwin TEM operating at $120 \mathrm{kV}$ under low-dose conditions (5 electrons / Å2). At similar defocus values $(-1.0 \mu \mathrm{m}$ to $-3.0 \mu \mathrm{m}$ ), images of ice-embedded DLPs prepared over holes (Figure 3C) exhibited less visual contrast than those prepared on cryo-SiN (Figure 3D). Also, molecular adaptors coating the surface of the cryo-SiN microchips could be seen in the background of the images. We could not visually distinguish these adaptors in the background of specimens prepared using holey carbon films. Frozen-hydrated samples prepared without antibodies did not yield specimens with appreciable quantities of rotavirus particles, indicating that antibodies are required to recruit DLPs -- and that they are present in the specimens prepared over holes. Individual DLPs selected from images acquired for both specimens using the SPIDER software 
Citation: Tanner JR, Demmert AC, Dukes MJ, Melanson LA, McDonald SM, et al. Cryo-SiN - An Alternative Substrate to Visualize Active Viral Assemblies. J Analyt Molecul Tech 2013;1(1): 6.

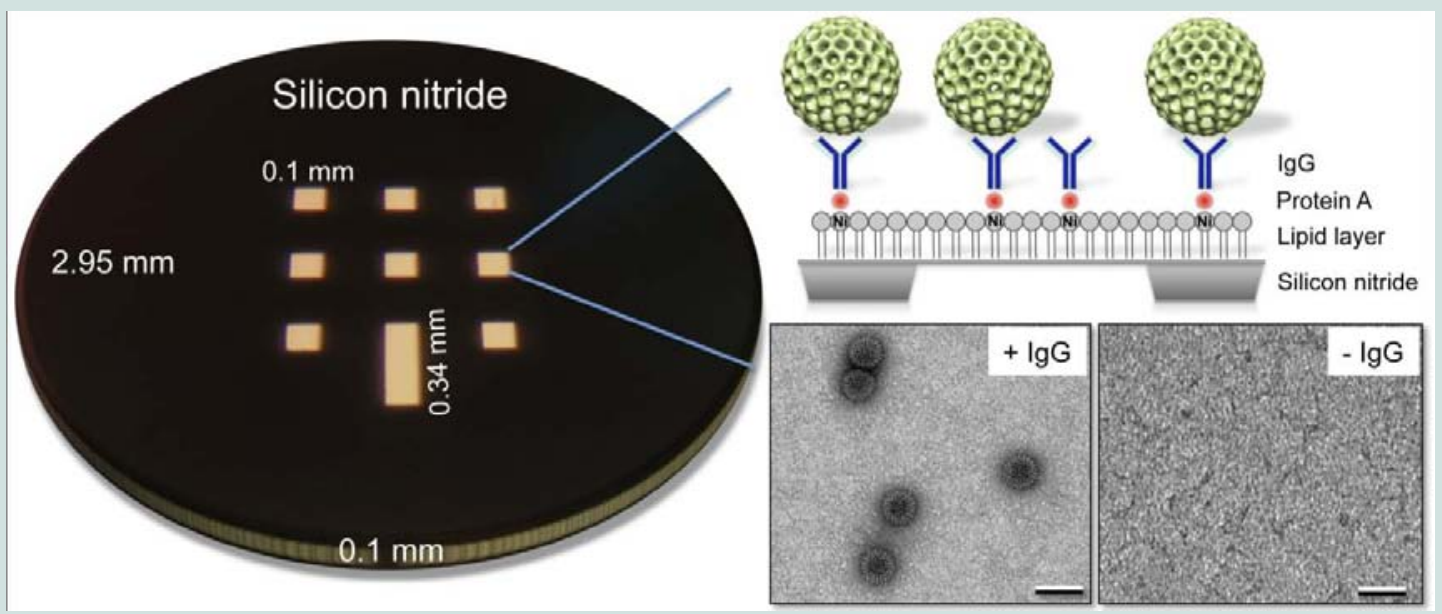

Figure 1: Affinity capture of rotavirus DLPs. SiN microchips primarily used in our analysis contain membranes etched down to $20-\mathrm{nm}$ thin regions at 9 discrete positions (left). SiN microchips were functionalized with Ni-NTA-containing lipid layers and used for the Affinity Capture functionalization process [11]. This procedure (top, right) entails coating SiN microchips with His-tagged protein A (red) and IgG antibodies (blue) that are used to recruit rotavirus DLPs (green). Representative images (bottom right) of negatively stained rotavirus DLPs samples prepared in the presence and absence of IgG adaptors. Scale bar is $100 \mathrm{~nm}$.

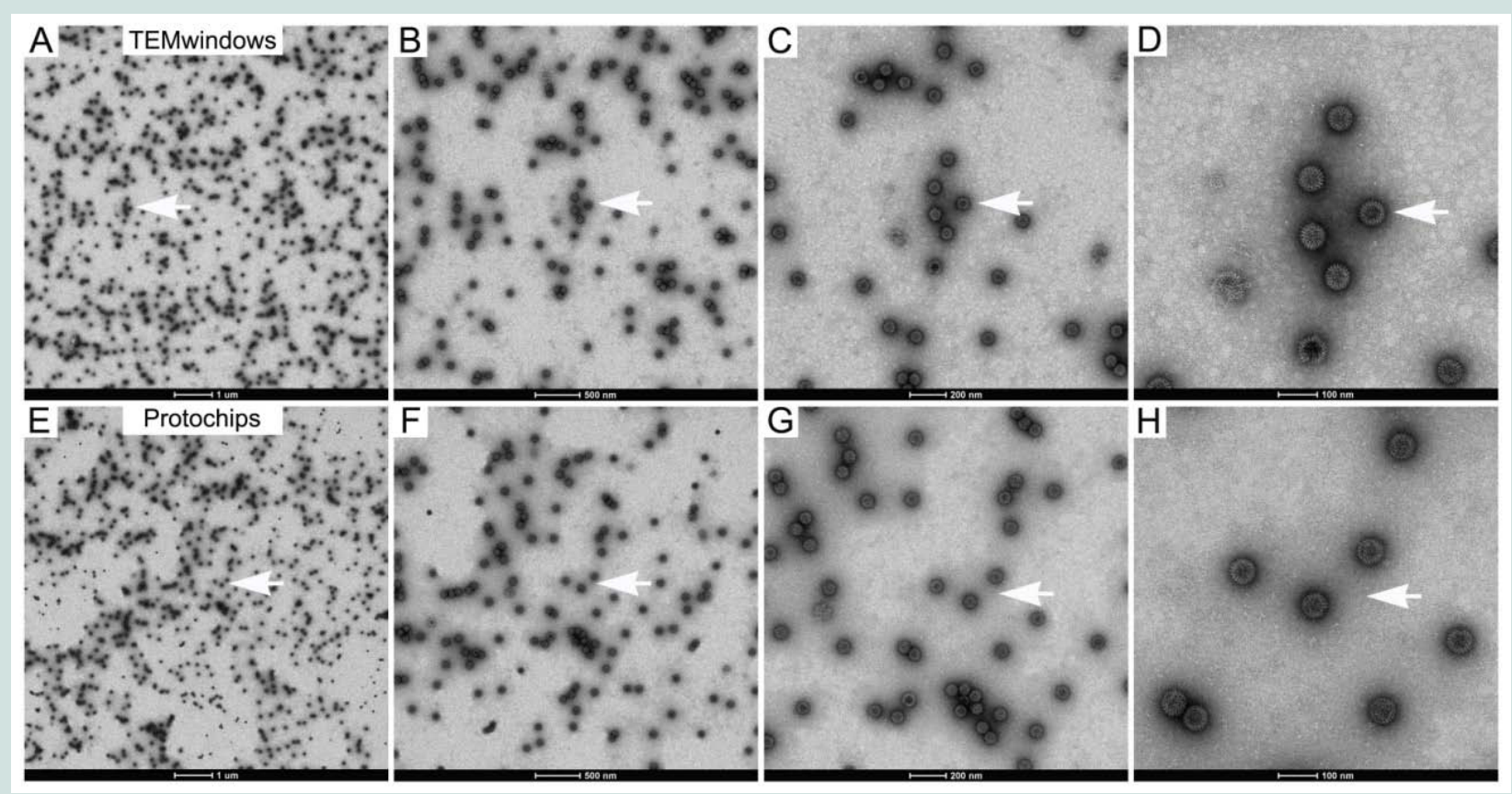

Figure 2: Images to indicate sample coverage on SiN microchips. Viral particles were well distributed on functionalized SiN microchips having 20-nm thick windows (A-D) provided by TEMwindows and 30-nm thick windows (E-H) provided by Protochips, Inc. Specimens were imaged at 4800× (A, E), 11,000× (B, F), $23,000 \times(\mathbf{C}, \mathbf{G})$, and $50,000 \times(\mathbf{D}, \mathbf{H})$. Clusters of the same particles are indicated across each magnification series by white arrows.

package [13].

\section{D Reconstructions reveal conserved capsids with variable cores}

Selected particle images of DLPs from specimens prepared on functionalized holey carbon film and cryo-SiN were exported into the RELION software package [14]. EM reconstructions were calculated for each type of specimen and included 572 particles for images of DLPs over holes (Figure 3E) and 38 particles for images of DLPs on cryo-SiN (Figure 3F). Using the RMEASURE program [16] the reconstruction calculated for DLPs in holes was approximately 2.4 $\mathrm{nm}$ while the reconstruction calculated for DLPs on cryo-SiN was estimated to be $2.5 \mathrm{~nm}$. As a control, we selected 40 random particles from specimens prepared on holey carbon film and used the same computing parameters. The resulting reconstruction was spherical and of proper dimensions, but lacked major features (data not shown). These results suggested that specimens prepared on cryoSiN could yield $3 \mathrm{D}$ reconstructions at a similar resolution as those prepared on holey carbon film, but using a fraction of the particles. Likewise, we could visualize more distinct densities in the interior of the cryo-SiN reconstructions (Figure $3 F$ ) versus those calculated 
Citation: Tanner JR, Demmert AC, Dukes MJ, Melanson LA, McDonald SM, et al. Cryo-SiN - An Alternative Substrate to Visualize Active Viral Assemblies. J Analyt Molecul Tech 2013;1(1): 6.
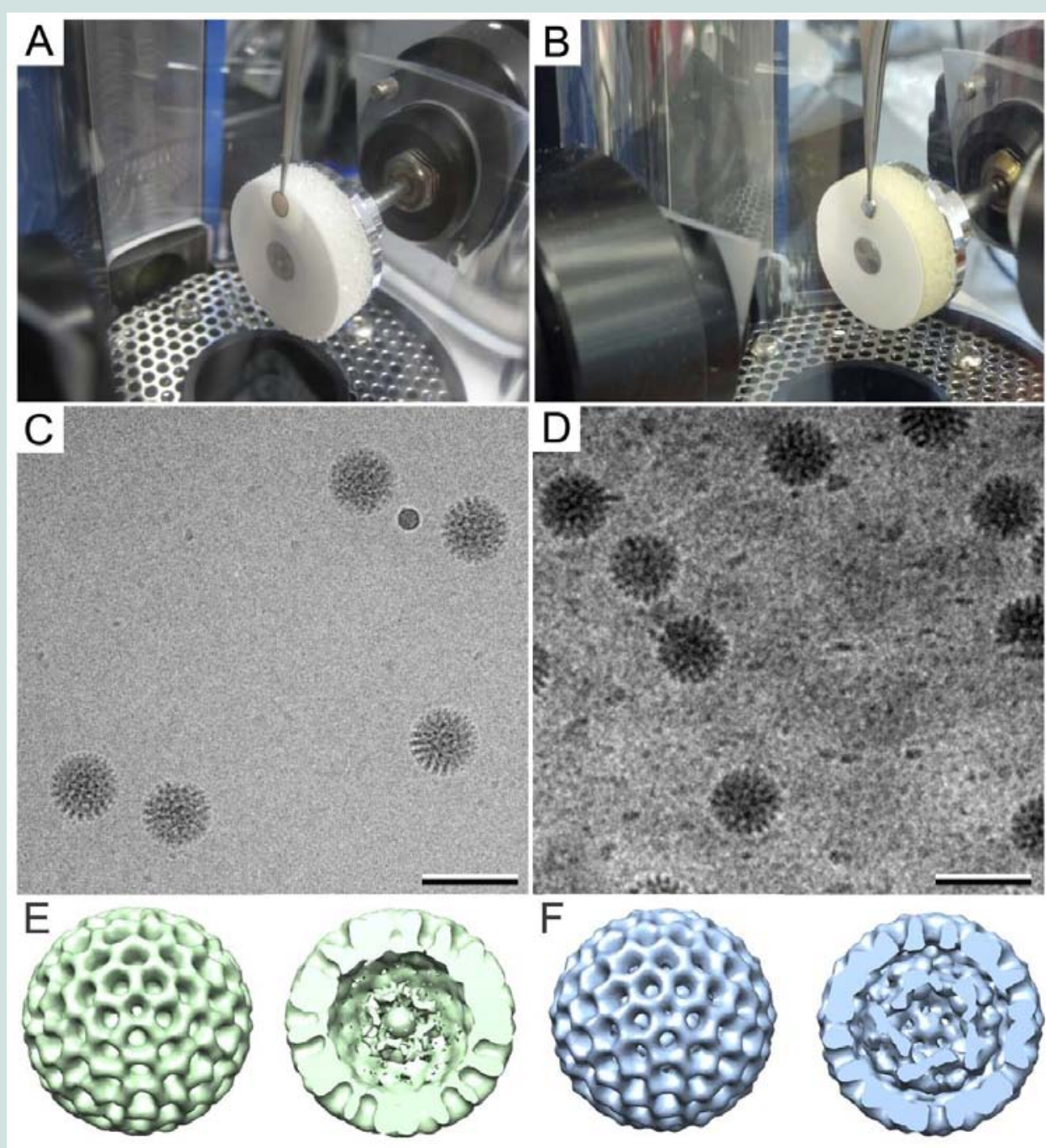

Figure 3: Vitrified inactive rotavirus DLPs. Cryo-EM specimens of rotavirus DLPs were prepared using a Gatan Cryoplunge ${ }^{\mathrm{TM}} 3$ with gentle-blot technology to rapidly plunge-freeze samples into liquid ethane. Both holey carbon Affinity grids $(\mathbf{A})$ and SiN microchips (B) were blotted from one side during the freezing process. Representative images indicate vitrified inactive DLPs can be similarly prepared on functionalized holey carbon Affinity grids (C) and cryo-SiN (D). Cross-sectional views of 3D reconstructions calculated from samples prepared on holey carbon Affinity grids (E) and cryo-SiN microchips (F). Reconstructions derived from cryo$\mathrm{SiN}$ samples show stronger density in the interior of the structures than reconstructions derived from holey carbon samples. Scale bar is $100 \mathrm{~nm}$. The diameter of each reconstruction is $\sim 80 \mathrm{~nm}$.

from DLPs on holey carbon films (Figure 3E).

\section{Transcribing DLPs captured while synthesizing mRNA}

Next, we compared the ability of the two substrates to host active viral processes using DLPs in the midst of synthesizing singlestranded mRNA transcripts. We produced specimens of DLPs induced to transcribe [11] implementing the same affinity capture and plunge freezing conditions that were used for inactive DLPs. Actively transcribing DLPs were prepared on functionalized holey carbon Affinity grids (Figure 4A) and on cryo-SiN (Figure 4B); and specimens were imaged using the same conditions described for the inactive DLPs. Particles from transcribing DLP specimens were selected using the SPIDER software package and imported into the RELION package. Implementing the same computational routines utilized for the inactive samples, we calculated $3 \mathrm{D}$ reconstructions for the transcribing DLPs. This data included 192 particles (Figure 4C) selected from the holey carbon Affinity grids and 100 particles from the functionalized cryo-SiN microchips (Figure 4D). Similar resolutions $(\sim 2.8 \mathrm{~nm})$ were obtained for comparable reconstructions having ordered outer capsids, although additional heterogeneous structures were statistically present in both cases. Again, stronger densities having more discrete features were present in the core of the reconstruction derived from cryo-SiN samples verses the reconstruction derived from holey carbon samples. This result is consistent with our findings for inactive DLPs prepared on the two substrates. Collectively, these results suggested that although both substrates could be used to prepare frozen-hydrated specimens of actively transcribing viral assemblies, cryo-SiN samples yielded 3D structures with well-defined features within their capsid interiors (Figure 4D). 


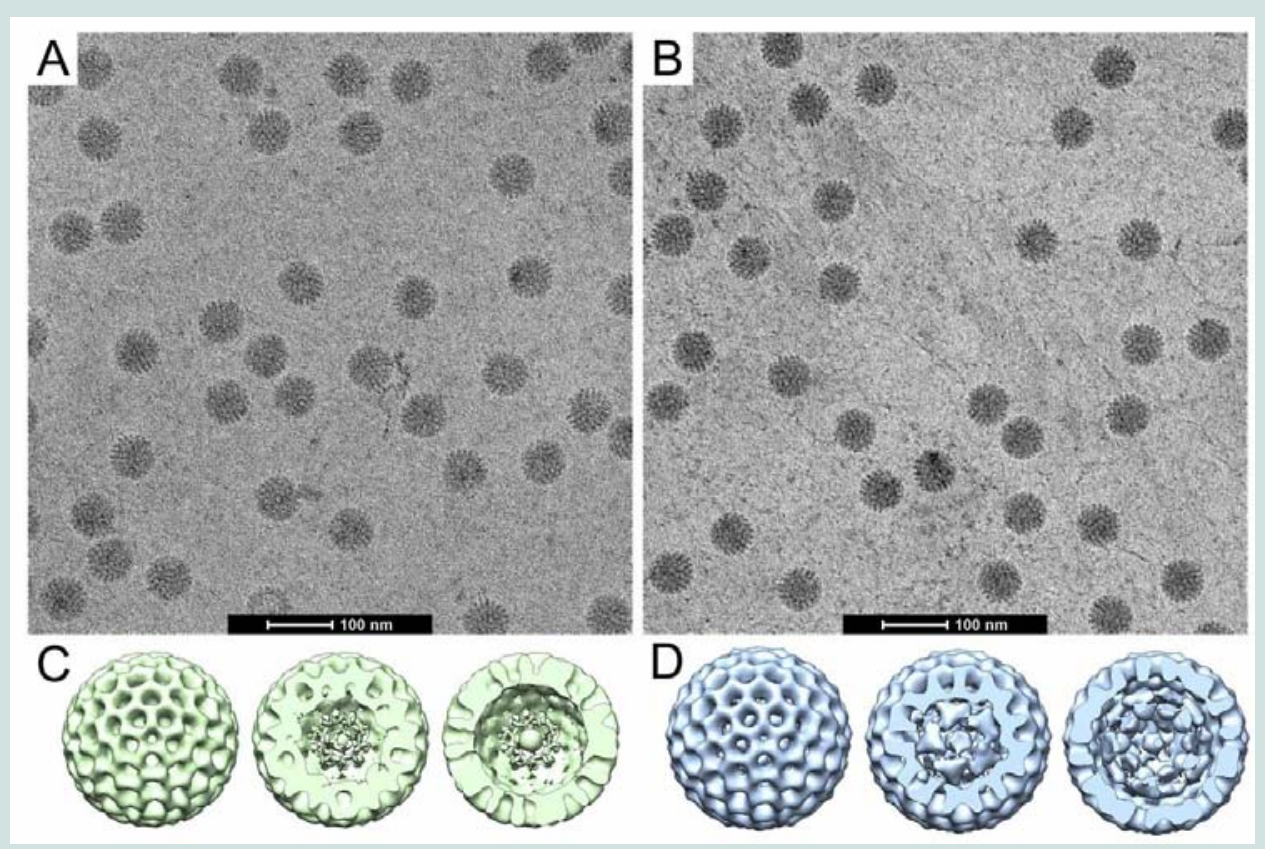

Figure 4: Vitrified transcriptionally active rotavirus DLPs. Representative images of transcriptionally active rotavirus DLPs vitrified on functionalized holey carbon Affinity grids (A) and cryo-SiN microchips (B). 3D reconstructions and corresponding cross-sections through each reconstruction indicate that a greater number of well defined features were present in transcribing particles prepared using cryo-SiN (D) in comparison to holey carbon grids (C). The diameter of each reconstruction is $\sim 80 \mathrm{~nm}$.

\section{Discussion}

The use of new materials, such as SiN, to prepare cryo-EM specimens can be beneficial due to their flatness, consistent production, and commercial availability. When comparing frozen hydrated biological specimens prepared on holey carbon films to cryo-SiN membranes, we noted key differences in the recorded images and downstream computational analyses. Consistent with our previously findings, functionalization steps and incubation reactions can be performed directly upon the SiN microchips [11] prior to plunge-freezing. Upon comparing images acquired on the same virus samples and under similar conditions, we determined that visual contrast was enhanced to some extent in viral assemblies prepared on cryo-SiN. This effect may be due to less charging in the cryo-SiN specimens during imaging or simply to our ability to produce thinner specimens on $\mathrm{SiN}$ membranes. We are currently investigating this topic on a deeper level as we strive to assess biological specimens at high-resolution. A high-resolution structure for the inactive rotavirus DLP capsid layer has been previously determined [15]. In our present work, the features and dimensions of frozen-hydrated inactive DLPs produced on holey carbon film and on cryo-SiN are consistent with the previously published structures regarding the architecture of the outer capsid. Similarly, the lack of major internal density within the capsid, as seen in inactive DLP specimens produced on holey carbon film, is consistent with previous findings [15]. Thus, DLP samples produced on holey carbon grids in our study served as adequate control specimens to ensure consistency in the use of reagents and techniques during specimen preparation.

With this in mind, DLP specimens produced on cryo-SiN revealed unique well defined features in the capsid interior, not previously seen in the published structure. We attribute this difference, in part, to the higher degree of visual contrast seen in the individual particles imaged on cryo-SiN (i.e., our ability to visualize particles more clearly in ice-embedded specimens may lead to better particle selection and alignment routines in subsequent processing steps). As a result, we could calculate $3 \mathrm{D}$ structures of DLPs prepared on cryo-SiN using a fraction of the images required for specimens prepared on holey carbon film. However, these procedures will need to be verified using other biological assemblies, including those that lack icosahedral symmetry. Future studies on inactive DLPs may shed light on the achievable spatial resolution for frozen hydrated biological specimens prepared on cryo-SiN.

Previous structural studies on transcribing DLPs also failed to define density in the internal cores [17]. Consistent with this work, highly-ordered transcribing DLPs imaged over holey carbon showed very little internal density. However, transcribing DLPs prepared on cryo-SiN contained strong internal density within DLPs having highly-ordered capsids. Therefore, we anticipate the use of the SiN substrates may potentially enhance our ability to assess structural features in cryo-EM virus specimens. This information could permit members of the virology community to examine active biological mechanism in a new way. Collectively, our findings suggest that silicon-based substrates may provide versatile new surfaces to view essential steps in viral processes. EM studies employing cryo-SiN may, therefore, offer unique insights for the development of antiretroviral therapies or vaccines to improve human health.

\section{References}

1. Taylor KA, Glaeser RM (2008) Retrospective on the early development of cryoelectron microscopy of macromolecules and a prospective on opportunities for the future. J Struct Biol 163: 214-223.

2. Brilot AF, Chen JZ, Cheng AC, Pan JH, Harrison SC, et al. (2012) Beam 
Citation: Tanner JR, Demmert AC, Dukes MJ, Melanson LA, McDonald SM, et al. Cryo-SiN - An Alternative Substrate to Visualize Active Viral Assemblies. J Analyt Molecul Tech 2013;1(1): 6.

\section{ISSN: 2474-1914}

induced motion of vitrified specimen on holey carbon film. J Struct Biol 177: 630-637.

3. Rhinow D, Kuhlbrandt W (2008) Electron cryo-microscopy of biological specimens on conductive titanium-silicon metal glass films. Ultramicroscopy 108: 698-705.

4. Yoshioka C, Carragher B, Potter CS (2010) Cryomesh (TM): A New Substrate for Cryo-Electron Microscopy. Microsc Microanal 16: 43-53.

5. Alloyeau D, Ding BQ, Ramasse Q, Kisielowski C, Lee Z, et al. (2011) Direct imaging and chemical analysis of unstained DNA origami performed with a transmission electron microscope. Chem Commun 47: 9375-9377.

6. Chen CC, Zhu C, White ER, Chiu CY, Scott MC, et al. (2013) Threedimensional imaging of dislocations in a nanoparticle at atomic resolution. Nature 496: 74-77.

7. Brown L, Hovden R, Huang P, Wojcik M, Muller DA, et al. (2012) Twinning and Twisting of Tri- and Bilayer Graphene, Nano Lett 12: 1609-1615.

8. Scott MC, Chen CC, Mecklenburg M, Zhu C, Xu R, et al. (2012) Electron tomography at 2.4-angstrom resolution. Nature 483: 444-447.

9. Kelly DF, Abeyrathne PD, Dukovski D, Walz T (2008) The Affinity Grid: a prefabricated EM grid for monolayer purification. J Mol Biol 382: 423-433.

10. Degen K, Dukes M, Tanner JR, Kelly DF (2012) The development of affinity capture devices-a nanoscale purification platform for biological in situ transmission electron microscopy. Rsc Adv 2: 2408-2412.

11. Gilmore BL, Tanner JR, McKell AO, Boudreaux CE, Dukes MJ, et al. (2013) Molecular Surveillance of Viral Processes Using Silicon Nitride Membranes. Micromachines 4: 90-102.

12. Gilmore BL, Showalter SP, Dukes MJ, Tanner JR, Demmert AC, et al. (2013) Visualizing viral assemblies in a nanoscale biosphere. Lab Chip 13: 216219.

13. Frank J, Radermacher M, Penczek P, Zhu J, Li Y, et al. (1996) SPIDER and WEB: processing and visualization of images in 3D electron microscopy and related fields. J Struct Biol 116: 190-199.

14. Scheres SH (2012) A Bayesian view on cryo-EM structure determination. J Mol Biol 415: 406-418.

15. Zhang X, Settembre E, Xu C, Dormitzer PR, Bellamy R, et al. (2008) Near-atomic resolution using electron cryomicroscopy and single-particle reconstruction. Proc Natl Acad Sci USA 105: 1867-1872.

16. Sousa D, Grigorieff $N$ (2007) Ab initio resolution measurement for single particle structures. J Struct Bio 157: 201-210.

17. Lawton JA, MK Estes, Prasad BV (1997) Three-dimensional visualization of mRNA release from actively transcribing rotavirus particles. Nat Struct Biol 4: 118-121.

\section{Acknowledgements}

The authors would like to thank Michael Friedlander as this work was sponsored by development funds to S.M.M. and D.F.K. from Virginia Tech. We appreciate the useful technical advice provided by James Roussie of TEMwindows, a division of SiMPore, Inc. and by John Damiano of Protochips, Inc. 